\title{
EQUIVALENCE OF TWO STRONG FORMS OF DETERMINACY
}

\section{ANDREAS BLASS}

ABSTRACT. Determinacy of all games of length $\omega$ with moves from $R$ is equivalent to determinacy of all games of length $\omega^{2}$ with moves from $\omega$.

The purpose of this note is to show that two apparently different ways of strengthening the axiom of determinacy lead to equivalent results. One way is to consider games in which there are uncountably many possible moves from each position. The other is to consider games in which each play has length an ordinal greater than $\omega$. We prove that determinacy for games with $2^{\boldsymbol{K}_{0}}$ moves from each position (and plays of length $\omega$ ) is equivalent to determinacy for games with plays of length $\omega^{2}$ (and $\kappa_{0}$ possible moves from each position).

For any set $A$ and any ordinal number $\alpha$, let $\operatorname{AD}(A, \alpha)$ be the assertion that one of the players has a winning strategy in every game of the following sort. The two players alternately select members of $A$ until, after $\alpha$ moves, they have produced a sequence (or play) $x=\langle x(\beta): \beta<\alpha\rangle$; the rules of the game are given by a certain set $G$ of $\alpha$-sequences from $A$, and the first player wins the play $x$ if $x \in G$. For more details see [1]. We shall prove the following result (in Zermelo-Fraenkel set theory without the axiom of choice).

Theorem. $\operatorname{AD}\left(\omega, \omega^{2}\right)$ is equivalent to $\operatorname{AD}(\mathbf{R}, \omega)$.

Here $\omega$ and $\omega^{2}$ have their usual meanings as ordinals, and $\mathbf{R}$ is the set of functions from $\omega$ to $\omega$; by the usual abuse of language, the members of $\mathbf{R}$ will be called reals. Before embarking on the proof of the theorem, we make a number of remarks, some for later use and some for general information.

1. If the cardinality $|A|$ of $A$ is $\leq|B|$ and if $\alpha \leq \beta$, then $\operatorname{AD}(B, \beta)$ implies $\operatorname{AD}(A, \alpha)$. Thus, our theorem remains true if $\mathbf{R}$ is taken to be the set of analysts' reals (rather than set-theorists' reals). Also, each of the propositions $\mathrm{AD}\left(\omega, \omega^{2}\right)$ and $\mathrm{AD}(\mathbf{R}, \omega)$ implies $\operatorname{AD}(\omega, \omega)$, the ordinary axiom of determinacy, AD.

2. Let $\mathrm{AC}(A)$ be the special case of the axiom of choice which

Received by the editors July 15, 1974.

AMS (MOS) subject classifications (1970). Primary 04A20, 04 A25. 
asserts that any set of $|A|$ or fewer nonempty subsets of $A$ admits a choice function. Then $\operatorname{AC}(A)$ is equivalent to $\operatorname{AD}(A, 2)$. See [1].

3. Any game over $\mathbf{R}$ of length $a$ can be viewed as a game of length $\omega$ - $\alpha$ over $\omega$. One move in the former game (choosing a real, i.e. an $\omega$ sequence of natural numbers) is represented by $\omega$ moves in the latter game. This possibility was noted by Mycielski [1]. It immediately implies half of our theorem: $\operatorname{AD}\left(\omega, \omega^{2}\right)$ implies $\operatorname{AD}(\mathbf{R}, \omega)$.

The preceding three remarks give us the implications in the following diagram:

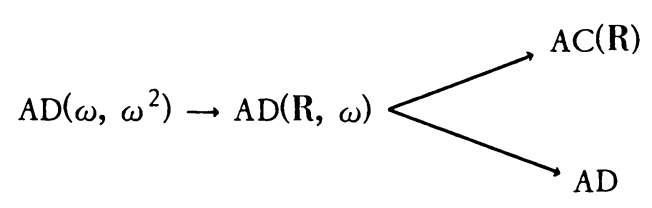

5. AC(R), being a consequence of the axiom of choice, cannot imply $A D$ which contradicts the axiom of choice.

6. AD does not imply $A C(R)$. This result (which, I am told, was first proved by Solovay) follows from the facts that (a) if $A D$ holds then it continues to hold in $\operatorname{HOD}(\mathbf{R})$, and (b) if $\operatorname{AC}(\mathbf{R})$ holds in $\operatorname{HOD}(\mathbf{R})$ then so does the full axiom of choice.

7. The conjunction of $A C(R)$ and $A D$ is equivalent to $\operatorname{AD}(\omega, \alpha)$ for each a satisfying $\omega \cdot 2 \leq a<\omega^{2}$. I do not know whether this conjunction implies $\operatorname{AD}\left(\omega, \omega^{2}\right)$, but it seems very unlikely.

At the referee's suggestion, we note that, if $\alpha+\beta<\boldsymbol{N}_{1}$, then $\operatorname{AD}(\mathbf{R}, \max (\alpha, 1+\beta))$ implies $\operatorname{AD}(\mathbf{R}, \alpha+\beta)$. The proof, like that of remark 7 , is based on considering, for any game $G$ of length $\alpha+\beta$, the game $G^{\prime}$ of length $\alpha$ in which each player tries to reach a position in $G$ such that he has a winning strategy for the remaining $\beta$ moves in $G$. (With some caution in avoiding the axiom of choice, one can replace $1+\beta$ with $\beta$ unless $\beta=1$.)

8. $\operatorname{AD}\left(\omega, \boldsymbol{\kappa}_{1}\right)$ is inconsistent with ZF. J. Mycielski has pointed out that his proof of this fact in [1] is incorrect but that the result is neverthe less true because $\operatorname{AD}\left(\omega, \boldsymbol{x}_{1}\right)$ implies $\operatorname{AD}\left(\boldsymbol{x}_{1}, \omega\right)$ which is false in $\mathrm{ZF}[1$, p. 217]. The implication is established by noting that, in games of length $\boldsymbol{N}_{1}$, a player can code a countable ordinal $a$ with a sequence of a zeros followed by a one.

Mycielski conjectured [1] that the axiom " $A D(\omega, \alpha)$ for all countable $a^{\prime \prime}$ is consistent with $\mathrm{ZF}$, and Solovay has remarked that this axiom might be true in the smallest transitive model of $\mathrm{ZF}$ containing all $\omega$-sequences of ordinals.

Proof of the theorem. Because of Remark 3, we need only prove that $\operatorname{AD}(\mathbf{R}, \omega)$ implies $\operatorname{AD}\left(\omega, \omega^{2}\right)$. So assume $\operatorname{AD}(\mathbf{R}, \omega)$ and its consequences 
$\mathrm{AD}$ and $\mathrm{AC}(\mathbf{R})$, and let $G$ be a game of length $\omega^{2}$ over $\omega$. Think of each play of $G$ as consisting of $\omega$ blocks of $\omega$ consecutive moves each.

Define an auxiliary game $G^{\prime}$ which is played just like $G$ except that, at the beginning of each block, player I must reveal the strategy he intends to follow during that block. Thus $G^{\prime}$ also consists of $\omega$ blocks, but each block consists of just two moves: I reveals a strategy $\sigma$ and II gives his list $q$ of $\omega$ moves. There is no need for I to actually make his $\omega$ moves, for they are determined by $\sigma$ and $q$. Clearly, each of II's moves in $G^{\prime}$ is a real. Each of I's moves $\sigma$ is a function from finite sequences of natural numbers to natural numbers; by Gödel numbering the finite sequences, we can think of $\sigma$ as a real. Thus $G^{\prime}$ is a game of length $\omega$ over $\mathbf{R}$, so it is determined.

$G^{\prime}$, compared to $G$, clearly puts player I at a disadvantage, for he must reveal his strategy for each block beforehand. Thus, if 1 has a winning strategy in $G^{\prime}$ then he also has one in $G$ (namely to use, rather than reveal, the strategies $\sigma$ supplied by his winning strategy in $G^{\prime}$ ). Now suppose II has a winning strategy $\tau$ in $G^{\prime}$; we shall produce a winning strategy for II in $G$, and this will finish the proof.

Consider all the plays $x$ of $G$ (functions from $\omega^{2}$ to $\omega$ ) that can arise when the players play $G^{\prime}$ and II uses $\tau$. All these sequences $x$ and all their initial segments of limit or zero length $(\omega \cdot n, n$ finite) will be called possibilities. Of course, all possibilities of length $\omega^{2}$ are wins for II, because $\tau$ is a winning strategy. For any possibility $y$ of length $\omega \cdot \gamma$ $(\gamma \leq \omega)$, there is a sequence $\Sigma=\left\langle\sigma_{\beta}: \beta<\gamma\right\rangle$ of moves of $I$ in $G^{\prime}$ such that $y$ results when I plays $\Sigma$ and II uses $\tau$. We then say that $\Sigma$ leads to $y$.

Given a possibility $y$ of length $\omega \cdot n<\omega^{2}$ and a sequence $\Sigma=$ $\left\langle\sigma_{0}, \cdots, \sigma_{n-1}\right\rangle$ leading to it, consider the one-term extensions $\Sigma^{\prime}=$ $\left\langle\sigma_{0}, \cdots, \sigma_{n-1}, \sigma_{n}\right\rangle$ of $\Sigma$ and the extensions $y^{\prime}$ of $y$ to which they lead. Several choices of $\Sigma^{\prime}$ may lead to the same $y^{\prime}$. For each $\Sigma, y$, and $y^{\prime}$ as above, we select a particular one-term extension $\Sigma^{\prime}$ of $\Sigma$ that leads to $y^{\prime}$, and we call these selected extensions (for all possible $y^{\prime}$ ) the standard extensions of $\Sigma$. Notice that the selection is possible because of $A C(R)$.

We will associate to certain possibilities $y$ specific sequences $\Sigma(y)$ leading to $y$. We proceed by induction on the length of $y$. To the empty possibility we associate the empty sequence. If we have associated $\Sigma(y)$ to $y$ and if some one-term extension of $\Sigma(y)$ leads to $y^{\prime}$ then we associate to $y^{\prime}$ the unique standard extension of $\Sigma(y)$ that leads to $y^{\prime}$. Notice that, if $\Sigma(z)$ is defined and $y$ is an initial segment of $z$, then $\Sigma(y)$ is defined and an initial segment of $\Sigma(z)$. Thus, if $y$ is a play of $G$ of the full length $\omega^{2}$, and all its initial segments $z$ of limit length have $\Sigma(z)$ defined, then these $\Sigma(z)$ 's are initial segments of a single $\Sigma$ which leads to $y$. It follows that $y$ is a possibility and therefore a win for II. 
We have shown that II wins all plays $y$ of $G$ whose initial segments $z$ of limit length have $\Sigma(z)$ defined. Our aim is to find a strategy for II in $G$ which guarantees that the play will have this property. It will suffice to show that, if $z$ has length $\omega \cdot n$ (i.e. $z$ is a play of the first $n$ blocks of $G$ ) and $\Sigma(z)$ is defined, then there is a strategy $r_{z}$ for II in the next block which will guarantee that, no matter what I does in that block, the play $z^{\prime}$ of the first $n+1$ blocks has $\Sigma\left(z^{\prime}\right)$ defined. For, if this is proved, then we can select one such $\tau_{z}$ for each such $z$ (by $A C(R)$ ) and build a winning strategy for II in $G$ by having II use the selected $\tau_{z}$ in the next block after $z$ has been played. It is clear by induction that when II uses this strategy, all the plays $z$ that can arise have $\Sigma(z)$ defined, so II will win.

It remains only to prove the existence of $\tau_{z}$. Consider any $z$ for which $\Sigma(z)=\left\langle\sigma_{0}, \ldots, \sigma_{n-1}\right\rangle$ is defined, and let

$$
G_{z}=\{q \in \mathbf{R}: \Sigma(z \text { concatenated with } q) \text { is not defined }\}
$$

$G_{z}$ is a game of length $\omega$ over $\omega$, so to show that II has a winning strategy (which will be the required $\tau_{z}$ ) we need only show that I does not have one and then invoke AD. So let $\sigma$ be any strategy for I in $G_{z}$. If I plays $\left\langle\sigma_{0}, \ldots, \sigma_{n-1}, \sigma\right\rangle$ in $G^{\prime}$, player II, using $\tau$, will respond with $n$ moves that result in $z$ (because $\Sigma(z)$ leads to $z$ ) and one more move $p$ (the reply to I's last move $\sigma$ ). If $q$ is the result of I using $\sigma$ against $p$, then, by definition, $\left\langle\sigma_{0}, \ldots, \sigma_{n-1}, \sigma\right\rangle$ leads to $z$ concatenated with $q$. But then $\Sigma(z$ concatenated with $q)$ is defined. This means that, in $G_{z}$, if I uses $\sigma$ and II plays $p$, then II wins; so $\sigma$ is not a winning strategy for I in $G_{z}$.

By trivial modifications of the preceding proof, one can obtain the following generalization.

Theorem. For every countable limit ordinal $\alpha, \operatorname{AD}(\omega, \omega \cdot \alpha)$ is equivalent to $\mathrm{AD}(\mathbf{R}, \alpha)$.

(The restriction to countable $\alpha$ is irrelevant because $\operatorname{AD}(\omega, \omega \cdot a)$ and $\operatorname{AD}(\mathbf{R}, a)$ are both false by Remark 8 if $\alpha$ is uncountable.)

After submitting this paper, the author learned that this theorem had already been proved by Jan Mycielski (unpublished) in 1967.

\section{REFERENCES}

1. J. Mycielski, $O_{n}$ the axiom of determinateness. I, II, Fund. Math. 53 (1963/ 64), 205-224; ibid. 59 (1966), 203-212. MR 28\#4991; 35 \#1489.

DEPARTMENT OF MATHEMATICS, UNIVERSITY OF MICHIGAN, ANN ARBOR, MICHIGAN 48104 